\title{
Core excitons and conduction-band structures in orthorhombic GeS, GeSe, SnS, and SnSe single crystals
}

\author{
M. Taniguchi, ${ }^{*}$ R. L. Johnson, ${ }^{\dagger}{ }^{J}$. Ghijsen, ${ }^{\ddagger}$ and M. Cardona \\ Max-Planck-Institut für Festkörperforschung, Heisenbergstrasse 1, Postfach 8006 65, D-7000 Stuttgart 80, \\ Federal Republic of Germany \\ (Received 4 December 1989; revised manuscript received 9 April 1990)
}

\begin{abstract}
Photoelectron partial-yield and constant-initial-state (CIS) spectra of the layered-semiconductor $\mathrm{GeS}, \mathrm{GeSe}, \mathrm{SnS}_{0.9} \mathrm{Se}_{0.1}$, and $\mathrm{SnSe}$ single crystals were investigated with use of synchrotron radiation. The cation $d$ core (Ge $3 d$ and Sn $4 d$ ) yield spectra show intense and sharp doublets due to cationderived core excitons. The CIS spectra exhibit strong resonance due to an interference between the direct-recombination process of the core excitons and the direct-excitation process of the valence electrons. The strong resonance is characteristic of core excitons with a long lifetime and a fairly localized nature. The anion $p$ and $d$ core (S $2 p$ and Se $3 d$ ) yield spectra are much broader than the cation $d$ core spectra and reflect the density of states (DOS) of the conduction bands. Energies of the experimental DOS peaks compare favorably with conduction-band features derived from interband optical-absorption spectra.
\end{abstract}

\section{INTRODUCTION}

The orthorhombic IV-VI compounds (GeS, GeSe, SnS, and $\mathrm{SnSe}$ ) have interesting electronic and optical properties and have been the subject of numerous investigations. The object of most of the work was to obtain information about the dielectric function and the band structure of these materials. A wide variety of techniques have been used including photoemission, ${ }^{1-3}$ energy-loss spectroscopy, ${ }^{4}$ reflectivity measurements, ${ }^{5}$ and band-structure calculations. ${ }^{2,6-8}$ In this paper, we concentrate on the corelevel excitations and conduction-band structures.

The layerlike orthorhombic chalcogenides all crystallize in the GeS structure which belongs to the orthorhombic space group $D_{2 h}^{16}(P n m a)$ and is closely related to the black phosphorus structure. The primitive cell contains eight atoms and covers two adjacent double layers as shown in Fig. 1(a). The atoms in a single layer are joined to three nearest neighbors by covalent bonds which form zigzag chains along the $b$ axis. All these crystals cleave exceptionally easily in the $a-b$ plane since van der Waals bonding predominates along the $c$ axis. The anisotropic crystal structure implies that significant differences are to be expected among the optical spectra with an electric vector parallel to the $a, b$, and $c$ directions.

Optical spectra due to the valence-electron excitation are related to the joint density of states (DOS) of the valence and conduction bands. On the other hand, because the initial core states have well-defined symmetry and negligibly small dispersion, core-electron excitation spectra provide not only information about the energies of flat regions of the conduction bands but also their symmetry and details of the electron-core-hole interaction. Previously, the $\mathrm{Sn} 4 d$ core reflectance spectrum of $\mathrm{SnS}$ (Ref. 5) was measured in the $a-b$ crystal plane. The dou- blet structure observed near the $4 d$ core threshold was interpreted taking the electron-core-hole interaction into account. However, the energy separation $(0.85 \mathrm{eV}) \mathrm{did}$ not agree with the $\mathrm{Sn} 4 d$ spin-orbit splitting $(1.08 \mathrm{eV})$ determined from photoemission measurements and this discrepancy beyond the experimental accuracy was not resolved.

We report here a systematic study of core excitons and conduction-band structures of $\mathrm{GeS}, \mathrm{GeSe}, \mathrm{SnS}_{0.9} \mathrm{Se}_{0.1}$, and $\mathrm{SnSe}$ single crystals using linearly polarized synchrotron radiation. The partial-yield spectra due to cation $d$ core excitations show intense and sharp coreexciton peaks near the core absorption thresholds with weak anisotropy in the $a-b$ crystal plane. The constantinitial-state (CIS) spectra elucidate the decay process of the core excitons, and indicate that the excitons are fairly localized. The anion $p$ (or $d$ ) core yield spectra reflect the conduction-band DOS. These results are analyzed and compared with band-structure calculations.

\section{EXPERIMENTAL DETAILS}

Experiments were performed at the Flipper II beamline in Hamburger Synchrotronstrahlungslabor (HASYLAB) at Deutsches Elektronen-Synchrotron DESY, Hamburg, ${ }^{9}$ using synchrotron radiation from the DORIS (DoppelRing Speicheranlage) storage ring. A combination of a plane grating monochromator (Flipper II) and a doublestage cylindrical mirror analyzer (PHI 25-260AR) was used to obtain the partial-yield and CIS spectra as well as the angle-integrated photoemission spectra of core levels and valence bands. Binding energies of the core-level and valence-band spectra were defined with respect to the valence-band maximum. The resolution of the monochromator was set to be $60 \mathrm{meV}$ at $25 \mathrm{eV}$. The GeS, $\mathrm{GeSe}, \mathrm{SnS}_{0.9} \mathrm{Se}_{0.1}$, and $\mathrm{SnSe}$ samples were undoped single crystals grown by the vacuum sublimation method. ${ }^{10}$ 
The orientation and lattice constants of these crystals were checked by $x$-ray diffraction. Surfaces for the measurements parallel to the $a-b$ crystal plane were prepared by cleaving under ultrahigh vacuum below $2 \times 10^{-10}$ mbar. The signal from a Au photodiode was used as a reference to normalize the partial-yield and CIS spectra, taking its absorption coefficients into account.

\section{RESULTS AND DISCUSSION}

In the IV-VI compound semiconductors GeS, GeSe, $\mathrm{SnS}$, and SnSe, the conduction bands are mainly derived
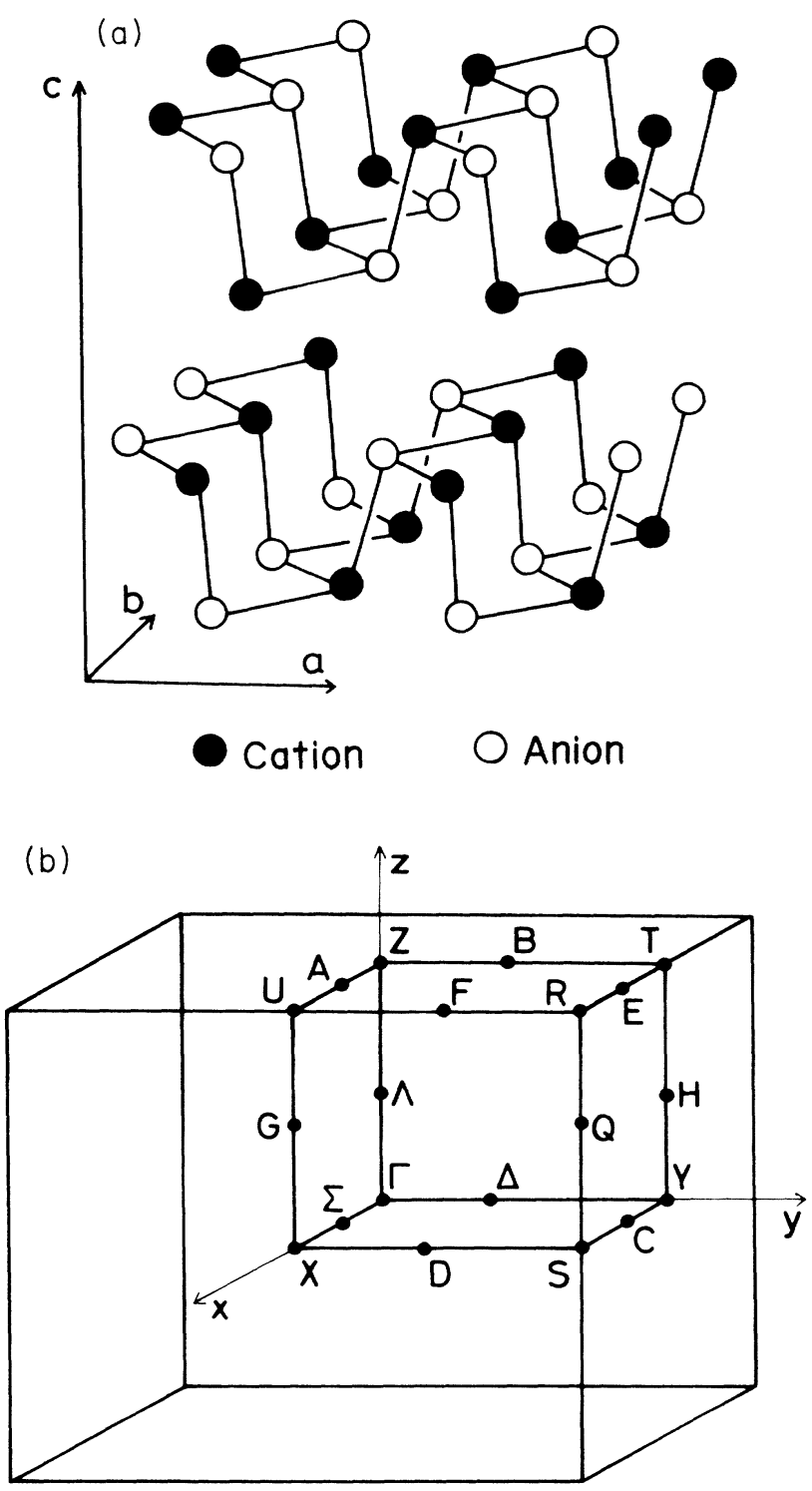

FIG. 1. (a) The germanium sulfide crystal structure. The unit cell is defined by $a, b$, and $c$ axes, where $a>b$ and $c$ is perpendicular to the cleavage plane. (b) Brillouin zone of the crystal. The $x, y$, and $z$ directions correspond to the $c, b$, and $a$ crystallographic axes.

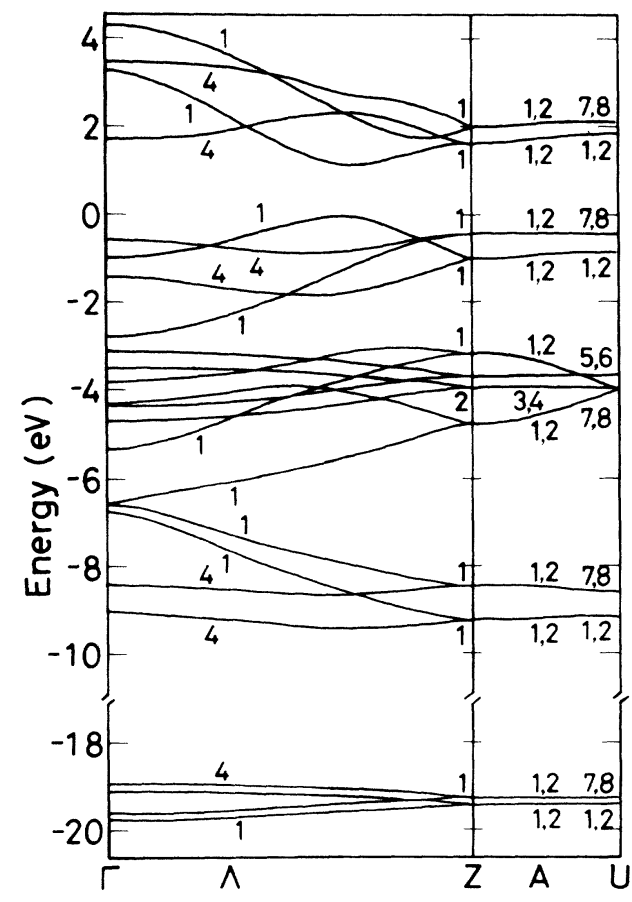

FIG. 2. Energy bands of GeS calculated using an empiricalpseudopotential method (Ref. 2).

from the cation (Ge $4 s^{2} 4 p^{2}$ and $\left.\mathrm{Sn} 5 s^{2} 5 p^{2}\right) p$ states with a smaller contribution from the anion (S $3 s^{2} 3 p^{4}$ and $\mathrm{Se}$ $\left.4 s^{2} 4 p^{4}\right) p$ states, because of the difference in valenceelectron configurations between the cation and anion

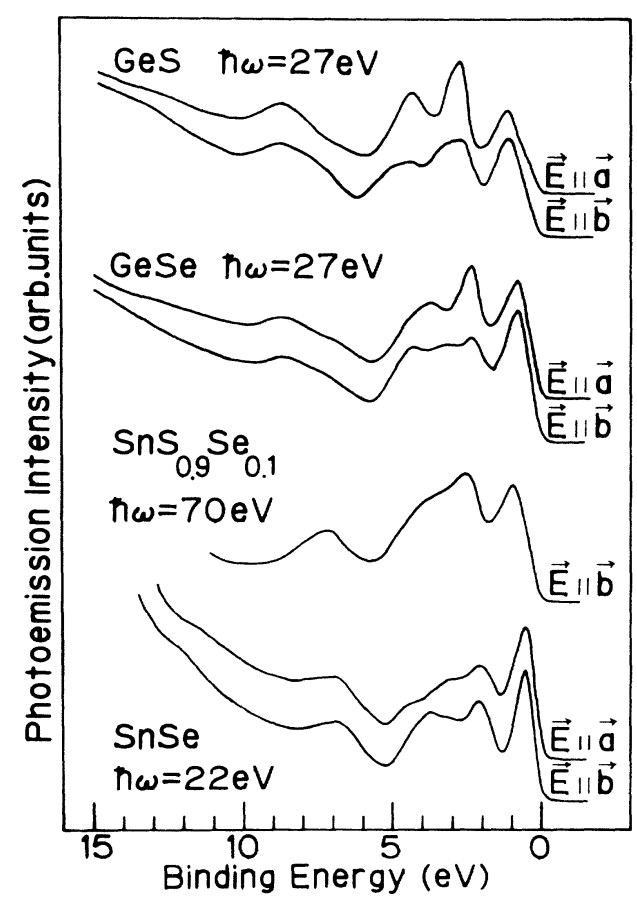

FIG. 3. Valence-band photoemission spectra of GeS, GeSe, $\mathrm{SnS}_{0.9} \mathrm{Se}_{0.1}$, and $\mathrm{SnSe}$ single crystals. The binding energy is defined with respect to the valence-band maximum. $\hbar \omega$ represents excitation photon energy. 
(e.g., $\mathrm{Ge}^{2+} 4 s^{2}, \mathrm{~S}^{2-} 3 s^{2} 3 p^{6}$ ) and the bonding ionicity of the crystals. The admixture between the $p$ and $s$ states in conduction bands is fairly small. This is reflected in the small dispersion of the cation and anion-derived $s$ valence bands over the whole Brillouin zone. ${ }^{2}$ Moreover, the conduction-band dispersions along the $Z-A-U$, $\Gamma-\Sigma-X, Y-C-S$, and $T-E-R$ lines perpendicular to the $a-b$ crystal plane are particularly small, reflecting the two-dimensional nature of these crystals [see Brillouin zone in Fig. 1(b) and energy-band structure in Fig. 2].

The valence bands can be divided into three regions (see photoemission spectra in Fig. 3). The first region extending from 0 - to $6-\mathrm{eV}$ binding energy is predominantly due to the bonding combination of the cation and anion $p$ electrons ( $p$ bands), while two regions of bands at 6-10and at $12-14-\mathrm{eV}$ binding energies are due to the cation and anion $s$ electrons ( $s$ bands), respectively. The weak $\mathrm{S}$ $3 s$ bands observed at $12-14-\mathrm{eV}$ binding energies for $\mathrm{GeS}$ in Fig. 3 are located at $\sim 19-\mathrm{eV}$ binding energy in the energy-band calculations. ${ }^{2}$ General features of the band structure of $\mathrm{GeS}, \mathrm{GeSe}, \mathrm{SnS}$, and $\mathrm{SnSe}$ are very similar to one another and to those of black P. ${ }^{11}$

\section{A. GeS and GeSe}

The band structure of GeS in Fig. 2 has been calculated with the empirical-pseudopotential method. ${ }^{2}$ The direct fundamental gap is located along the $\Lambda$ line. The calculated energy gap of $1.2 \mathrm{eV}$ is smaller than the experimental value of $1.65 \mathrm{eV} .{ }^{12}$ The energies and dispersions of the valence bands have already been compared with angle-resolved photoemission data. ${ }^{2}$ The accuracy of the calculation was reported to be about $0.2 \mathrm{eV}$ near the top of valence bands and about $1 \mathrm{eV}$ around 6-eV binding energy.

The energy-band calculations of GeSe have been carried out using the pseudopotential method. ${ }^{6}$ On the basis of the theoretical band structure and selection rules for optical dipole transitions, main structures in the interband optical spectra were analyzed in detail. Gross features of band structures for GeSe are very similar to those for GeS.

\section{Cation core excitation spectra}

Figure 4 shows the partial-yield spectra of $\mathrm{GeS}$ and GeSe for $\mathbf{E} \| \mathbf{a}$ and $\mathbf{E} \| \mathbf{b}$ in the Ge $3 d$ core excitation region. The partial-yield spectra are known to be representative of the absorption spectrum in the core excitation region. ${ }^{13}$ In the figure, $\mathbf{E}$ stands for the polarization vector of the light. The vertical arrows show the energy positions of the Ge $3 d_{5 / 2}$ core absorption thresholds ( 31.10 $\mathrm{eV}$ for $\mathrm{GeS}$ and $30.55 \mathrm{eV}$ for GeSe), which have been estimated from the sum of the band-gap energy $[1.65 \mathrm{eV}$ for $\mathrm{GeS}$ (Ref. 12) and $1.08 \mathrm{eV}$ for GeSe (Ref. 14)] and the Ge $3 d$ core-level binding energies with respect to the valence-band maximum. The binding energies derived from the core-level photoemission spectra are 29.45 and $29.98 \mathrm{eV}$ for the $3 d_{5 / 2}$ and $3 d_{3 / 2}$ core levels of GeS and 29.47 and $30.01 \mathrm{eV}$ for those of GeSe, respectively.

The spectrum of GeS for $\mathbf{E} \| \mathbf{a}$ clearly shows four structures in the energy region from 30.5 to $32 \mathrm{eV}$. Above 32

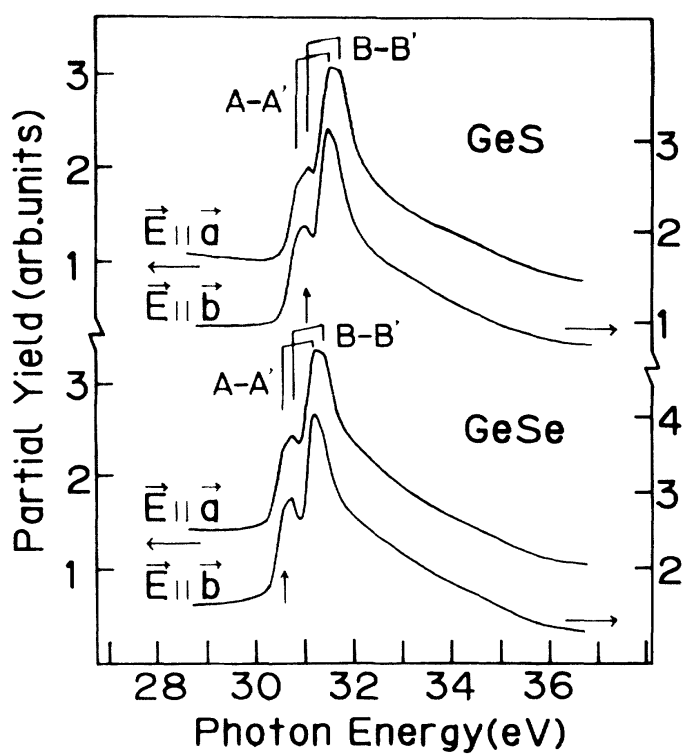

FIG. 4. Partial-yield spectra of GeS and GeSe for $\mathbf{E} \| \mathbf{a}$ and $\mathbf{E} \| \mathbf{b}$ in the Ge $3 d$ core excitation region.

eV, the spectrum exhibits no clear peak structures. The spectral shape for $\mathbf{E} \| \mathbf{b}$ is slightly different from that for $\mathbf{E} \| \mathbf{a}$ : a weak anisotropy is observed in the $a-b$ crystal plane concerning the dip between the structures $B$ and $\mathrm{A}^{\prime}$.

In order to resolve four structures near the Ge $3 d$ core threshold and to evaluate the energy positions of these structures, we have calculated the second energy derivatives of the partial-yield spectra. They are shown for GeS in Fig. 5. One notices that four structures are clearly resolved as negative peaks at $30.83(\mathrm{~A}), 31.05(\mathrm{~B}), 31.47$ $\left(\mathbf{A}^{\prime}\right)$, and $31.68 \mathrm{eV}\left(\mathbf{B}^{\prime}\right)$ for $\mathbf{E} \| \mathbf{a}[30.83(\mathbf{A}), 31.01(\mathbf{B})$, $31.45\left(\mathbf{A}^{\prime}\right)$, and $(31.65) \mathrm{eV}\left(\mathbf{B}^{\prime}\right)$ for $\left.\mathbf{E} \| \mathbf{b}\right]$. From the spectral shapes in Figs. 4 and 5, the relative intensities of the doublet structures $\mathbf{A}-\mathbf{A}^{\prime}$ and $\mathbf{B}-\mathbf{B}^{\prime}$ are found to deviate significantly from $6: 4$ statistical weight of the initial $3 d_{5 / 2}$ and $3 d_{3 / 2}$ core states. In addition, the splittings of these doublets $(\sim 0.64 \mathrm{eV})$ are slightly larger than the spinorbit splitting of the Ge $3 d$ core levels $(0.53 \mathrm{eV})$. The comparison between the spectra for $\mathbf{E} \| \mathbf{a}$ and $\mathbf{E} \| \mathbf{b}$ in Fig. 5 reveals a weak anisotropy in the $a-b$ crystal plane that is most apparent for the B-B' doublet.

Here, it is appropriate to review the general features of the DOS of the conduction bands for understanding the observed doublet structures $\mathbf{A}-\mathbf{A}^{\prime}$ and $\mathbf{B}-\mathbf{B}^{\prime}$, though theoretical DOS curves are not yet available. Taking the results of band calculation ${ }^{2}$ (Fig. 2) and the fundamental absorption experiments ${ }^{12}$ into account, we notice the relatively large dispersion of the lowest conduction band at the $\Lambda$ symmetry point. The free exciton associated with the direct fundamental gap at the $\Lambda$ symmetry point has a binding energy of $11.5 \mathrm{meV}$, corresponding to a reduced mass of $0.08 m_{0} \cdot{ }^{12}$ At $\sim 0.4 \mathrm{eV}$ above the conduction-band minimum, the quasi-two-dimensional bands with very small dispersion lie along the $Z-A-U$ 


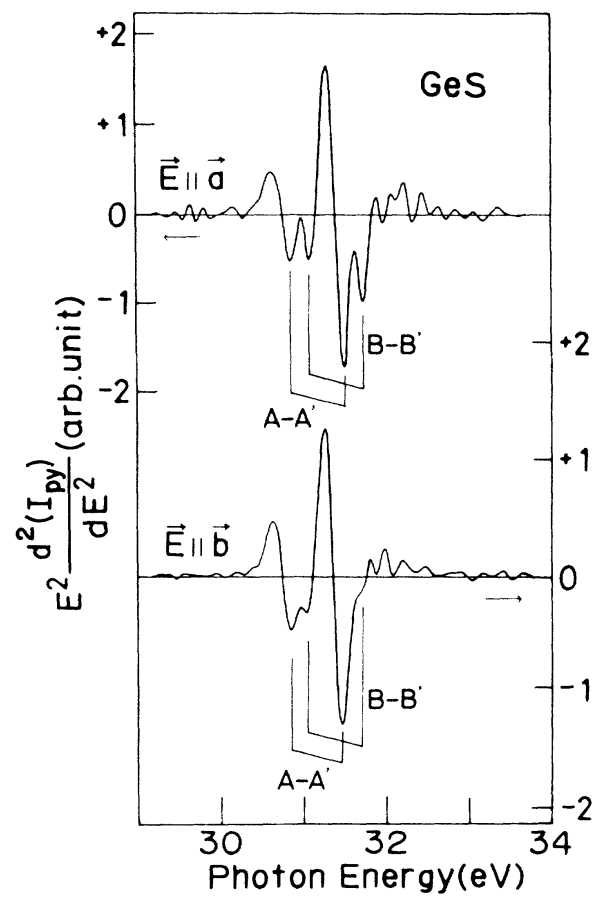

FIG. 5. Second-energy-derivative spectra of GeS for $\mathbf{E} \| \mathbf{a}$ and $\mathbf{E} \| \mathbf{b}$. The spectra have been numerically calculated by applying the least-squares fit to a quadratic function in the small energy region.

line. These results imply that the DOS of conduction bands of GeS increases gradually toward higher energy. At $\sim 0.4 \mathrm{eV}$ above the conduction-band minimum, the DOS curve has a first maximum, which stems from the two-dimensional bands with high DOS within a small energy interval. Such features can be recognized in the anion core excitation spectra shown later.

Comparing the partial-yield spectra with the DOS of conduction bands discussed above, the strong oscillator strength of the doublets $\mathrm{A}-\mathrm{A}^{\prime}$ and $\mathrm{B}-\mathrm{B}^{\prime}$ near the Ge $3 d$ core thresholds can be understood as originating from the quasi-two-dimensional conduction bands with very high DOS. The reversal of the relative intensity of these doublets and the deviation of the splitting energy of the doublets from the spin-orbit value can be interpreted as being due to electron-core-hole exchange interaction. ${ }^{15,16}$ Thus, we assign the doublet structures $\mathbf{A}-\mathbf{A}^{\prime}$ and $\mathbf{B}-\mathbf{B}^{\prime}$ as the spin-orbit doublets of the Ge $3 d$ core excitons associated with the two-dimensional conduction bands, the A$\mathbf{A}^{\prime}$ (B-B') separation corresponding to the core spin-orbit splitting. The weak anisotropy indicates that the core excitons, especially B-B', have a more extended character than a purely atomic excitation, though they are relatively tightly bound.

The spectra of GeSe exhibit structures at 30.56 (A), $30.77(B), 31.18\left(\mathbf{A}^{\prime}\right)$, and $31.39 \mathrm{eV}\left(\mathbf{B}^{\prime}\right)$ for $\mathbf{E} \| \mathbf{a}$ and 30.55 (A), $30.69(\mathbf{B}), 31.18\left(\mathbf{A}^{\prime}\right)$ and $(31.36) \mathrm{eV}\left(\mathbf{B}^{\prime}\right)$ for $\mathbf{E} \| \mathbf{b}$. The spectral features are basically the same as those of GeS regarding the reversal of intensities of the A- $\mathrm{A}^{\prime}$ and B-B' doublets and the slight difference in the energy split- tings of the doublet between the yield and core-level photoemission spectra and also the weak anisotropy in the $a$ $b$ crystal plane. Thus, the doublet structures $\mathbf{A}-\mathbf{A}^{\prime}$ and $\mathrm{B}^{-\mathrm{B}^{\prime}}$ in GeSe can be assigned to the Ge $3 d$ core-derived excitons.

\section{Anion core excitation spectra}

Figure 6 shows the partial-yield spectra of $\mathrm{GeS}$ and $\mathrm{GeSe}$ in the S $2 p$ and Se $3 d$ core excitation region. By comparing these spectra with the cation excitation spectra in Fig. 4, one notices the absence of intense and sharp peak structures near the core excitation thresholds shown by vertical arrows $(162.2 \mathrm{eV}$ for $\mathrm{GeS}$ and $54.40 \mathrm{eV}$ for $\mathrm{GeSe}$ ). All structures are observed as broad peaks. In addition, the relative intensities between the spin-orbit partners reflect the degeneracy of the initial core states (2:1 for the $2 p_{3 / 2}$ and $2 p_{1 / 2}$ core levels, and $6: 4$ for the $3 d_{5 / 2}$ and $3 d_{3 / 2}$ core levels). Therefore, it is reasonable to assume that the core excitation spectra map roughly the DOS of the conduction bands. We thus discuss the anion core yield spectra in terms of the one electron model, neglecting the electron-core-hole interaction.

The spectra of GeS for $\mathbf{E} \| \mathbf{a}$ and $\mathbf{E} \| \mathbf{b}$ show broad peaks at 162.9 (a) and $164.2 \mathrm{eV}\left(\mathrm{a}^{\prime}\right)$. The splitting energy between the $a$ and $a^{\prime}$ structures is almost equal to the spinorbit splitting of the $\mathrm{S} 2 p$ core level $(1.2 \mathrm{eV}) .{ }^{17}$ The structures $\mathbf{a}-\mathrm{a}^{\prime}$ are thus attributed to the corresponding spin-
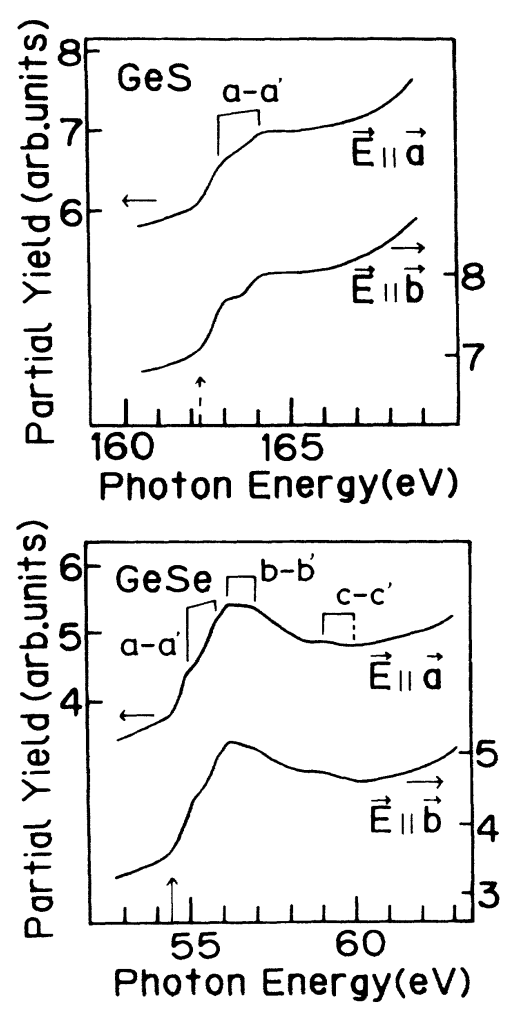

FIG. 6. Partial-yield spectra of GeS and GeSe for $\mathbf{E} \| \mathbf{a}$ and $\mathbf{E} \| \mathbf{b}$ in the $\mathbf{S} 2 p$ and Se $3 d$ core excitation regions. 
TABLE I. Peak energies in partial-yield spectra of GeS and interpretation of final states for optical transitions in terms of bandstructure calculation.

\begin{tabular}{|c|c|c|c|c|c|c|c|}
\hline & \multirow{3}{*}{ Structure } & \multicolumn{4}{|c|}{ Experiment } & \multicolumn{2}{|r|}{ Theory (Ref. 2) } \\
\hline & & Peak & hergy & \multicolumn{2}{|c|}{$\begin{array}{l}\text { Energy above the core } \\
\text { absorption threshold } \\
(\mathrm{eV})\end{array}$} & \multirow[t]{2}{*}{ Assignment } & \multirow[t]{2}{*}{$\begin{array}{c}\text { Energy above the } \\
\text { conduction-band minimum } \\
(\mathrm{eV})\end{array}$} \\
\hline & & $\mathbf{E} \| \mathbf{a}$ & $\mathbf{E} \| \mathbf{b}$ & $\mathbf{E} \| \mathbf{a}$ & $\mathbf{E} \| \mathbf{b}$ & & \\
\hline \multirow{4}{*}{$\begin{array}{l}\text { Ge } 3 d \text { core } \\
\text { excitation }\end{array}$} & A & 30.83 & 30.83 & -0.27 & -0.27 & \multirow{4}{*}{$\begin{array}{l}\text { core } \\
\text { excitons }\end{array}$} & \\
\hline & B & 31.05 & 31.01 & -0.05 & -0.09 & & \\
\hline & $\mathrm{A}^{\prime}$ & 31.47 & 31.45 & \multirow{5}{*}{0.7} & \multirow{5}{*}{0.7} & & \\
\hline & $\mathbf{B}^{\prime}$ & 31.68 & $(31.65)$ & & & & \\
\hline \multirow{3}{*}{$\begin{array}{l}\text { S } 2 p \text { core } \\
\text { excitation }\end{array}$} & $\mathbf{a}$ & 162.9 & 162.9 & & & $Z_{1}-A_{12}-U_{12}$ & $0.4-0.6$ \\
\hline & $a^{\prime}$ & 164.2 & 164.2 & & & $Z_{1}-A_{1.2}-U_{78}$ & $0.75-0.86$ \\
\hline & & & & & & $Y_{1}-C_{14}-S_{1.2}$ & $0.96-1.10$ \\
\hline
\end{tabular}

orbit doublet. We assign the structure a to the transition from the $2 p_{3 / 2}$ core level to the flat regions of conduction bands along the $Z_{1}-A_{1,2}-U_{1,2}, Z_{1}-A_{1,2}-U_{7,8}$, and $Y_{1}-C_{1,4}-S_{1,2}$ lines with very high DOS (Table I). This assignment is consistent with the interband spectra in the visible range. ${ }^{4}$ The valence-band spectra of GeS have shown the first $p$-band peak at $1.1-\mathrm{eV}$ binding energy with respect to the valence-band maximum (Fig. 3). Taking into account this binding energy and the band-gap energy of $1.65 \mathrm{eV}$ (Ref. 12) as well as the energy of the structure a $0.7 \mathrm{eV}$ above the core absorption threshold $(162.2 \mathrm{eV}){ }^{18}$ the interband transitions with strong oscillator strength are expected in the energy region around $3.5 \mathrm{eV}$. The imaginary parts of the experimental dielectric function $\left(\varepsilon_{2}\right)$ show prominent peaks at $3.1-3.7,{ }^{19}$ which were assigned to the interband transitions between twodimensional bands along the $Z-A-U$ and $Y-C-S$ lines. We thus conjecture that the first $p$ valence band and the main peak in the present partial-yield spectra are responsible for these initial and final states in the interband transitions.
The spectra of GeSe show structures at 55.0, 55.9, 56.2, 57.0, and $59.0 \mathrm{eV}$ for $\mathbf{E} \| \mathbf{a}$ and $55.0,55.9,56.2,57.1$, and $59.1 \mathrm{eV}$ for $\mathbf{E} \| \mathbf{b}$. Taking into account the initial $3 d$ corelevel splitting of $0.85 \mathrm{eV}$ calculated from the $3 d_{5 / 2}$ and $3 d_{3 / 2}$ core-level binding energies $(53.32$ and $54.17 \mathrm{eV}$, respectively), we have assigned these structures to the spin-orbit doublets in Fig. 6.

Using the same line of argument as for the $\mathrm{S} 2 p$ core spectra of GeS, one can estimate the energies of prominent peaks expected in the $\varepsilon_{2}$ spectra of GeSe. The simple consideration for the $0.8-\mathrm{eV}$ binding energy of the first $p$ valence-band peak (Fig. 3) and the band-gap energy of $1.08 \mathrm{eV},{ }^{14}$ as well as the energies of the structures $a, b$, and $\mathrm{c}(0.6,1.8$, and $4.7 \mathrm{eV}$ in Table II) above the core absorption threshold $(54.50 \mathrm{eV})$ implies peaks at 2.5, 3.7, and $6.5 \mathrm{eV}$ in the $\varepsilon_{2}$ spectra. The experimental $\varepsilon_{2}$ spectra indeed show prominent peaks at $2.45,3.45$, and $6.1 \mathrm{eV},{ }^{6}$ which have been assigned to the $U_{6}-U_{1}, U_{6}-U_{2}$, and $U_{1}$ $U_{6}$ transitions, in agreement with the evaluation above. The prominent peaks $a$ and $b$ in Fig. 6 are, thus, assigned to the transitions from the Se $3 d_{5 / 2}$ core level to the flat

TABLE II. Peak energies in partial-yield spectra of GeSe and interpretation of final states for optical transitions in terms of bandstructure calculation.

\begin{tabular}{|c|c|c|c|c|c|c|c|}
\hline & \multirow{3}{*}{ Structure } & \multicolumn{4}{|c|}{ Experiment } & \multicolumn{2}{|c|}{ Theory (Ref. 6) } \\
\hline & & \multicolumn{2}{|c|}{$\begin{array}{c}\text { Peak energy } \\
(\mathrm{eV})\end{array}$} & \multicolumn{2}{|c|}{$\begin{array}{c}\text { Energy above the core } \\
\text { absorption threshold } \\
(\mathrm{eV})\end{array}$} & \multirow[t]{2}{*}{ Assignment } & \multirow[t]{2}{*}{$\begin{array}{l}\text { Energy above the } \\
\text { conduction-band minimum } \\
(\mathrm{eV})\end{array}$} \\
\hline & & $\mathbf{E} \| \mathbf{a}$ & $\mathbf{E} \| \mathbf{b}$ & $\mathbf{E} \| \mathbf{a}$ & $\mathbf{E} \| \mathbf{b}$ & & \\
\hline \multirow{4}{*}{$\begin{array}{l}\text { Ge } 3 d \text { core } \\
\text { excitation }\end{array}$} & A & 30.56 & 30.55 & 0.01 & 0.00 & & \\
\hline & $\mathrm{B}$ & 30.77 & 30.69 & 0.22 & 0.14 & core & \\
\hline & $\mathbf{A}^{\prime}$ & 31.18 & 31.18 & & & excitons & \\
\hline & $\mathbf{B}^{\prime}$ & 31.39 & $(31.36)$ & & & & \\
\hline \multirow{5}{*}{$\begin{array}{l}\text { Se } 3 d \text { core } \\
\text { excitation }\end{array}$} & $\mathrm{a}$ & 55.0 & 55.0 & 0.6 & 0.6 & $Z_{1}-F_{1}-U_{1}$ & $0.56-0.58$ \\
\hline & $a^{\prime}$ & 55.9 & 55.9 & & & & \\
\hline & $\mathrm{b}$ & 56.2 & 56.2 & 1.8 & 1.8 & $Z_{2}-F_{2}-U_{2}$ & $1.55-1.62$ \\
\hline & $b^{\prime}$ & 57.0 & 57.1 & & & & \\
\hline & $\mathrm{c}$ & 59.0 & 59.1 & 4.6 & 4.7 & many flat regions & $4-5$ \\
\hline
\end{tabular}


regions of conduction bands along the $Z_{1}-F_{1}-U_{1}$ and $Z_{2}-F_{2}-U_{2}$ lines. $^{6}$ For structure (c), the assignment is difficult, since there are many flat bands around $4-5 \mathrm{eV}$ above the conduction-band minimum.

\section{B. SnS and SnSe}

Energy dispersion and DOS of valence bands of SnS have been calculated using empirical-pseudopotential methods, ${ }^{20}$ though theoretical calculation of the conduction bands is not yet available.

Energy-band structures of $\mathrm{SnSe}$ have been calculated on the basis of an empirical-pseudopotential approach. ${ }^{8}$ The result exhibits an indirect fundamental gap between the conduction-band minimum at the $\Gamma$ (or $\Sigma$ ) symmetry point and the valence-band maximum at the $\Lambda$ symmetry point. The calculated gap energy of $2.1 \mathrm{eV}$ is, however, much larger than the experimental value of $0.9 \mathrm{eV} .{ }^{21}$

Although there is limited accuracy in the theoretical results reported so far, we suppose that real band structures of $\mathrm{SnS}$ and $\mathrm{SnSe}$ would be very similar to each other.

\section{Cation excitation spectra}

Figure 7 shows the partial-yield spectra of $\mathrm{SnS}_{0.9} \mathrm{Se}_{0.1}$ and $\mathrm{SnSe}$ for $\mathbf{E} \| \mathbf{a}$ and $\mathbf{E} \| \mathbf{b}$ in the $\mathrm{Sn} 4 d$ core excitation region. Prominent sharp peaks are again observed in the region corresponding to excitations from the Sn $4 d$ core. Contrary to the case of $\mathrm{GeS}$ and $\mathrm{GeSe}$, one can recognize only three structures above the $\mathrm{Sn} 4 d$ core thresholds: a shoulder $\mathrm{A}$ and two strong peaks $\mathrm{B}$ and $\mathrm{A}^{\prime}$. In the figure, vertical arrows represent energy positions of the Sn $4 d_{5 / 2}$ core absorption thresholds $\left(25.4 \mathrm{eV}\right.$ for $\mathrm{SnS}_{0.9} \mathrm{Se}_{0.1}$ and $25.1 \mathrm{eV}$ for SnSe), obtained from the sum of the band-gap energies [1.1 eV for $\mathrm{SnS}$ (Ref. 22) and $0.9 \mathrm{eV}$ for $\mathrm{SnSe}$ (Ref. 21)] and the Sn $4 d$ core-level binding energies. The core-level binding energies have been determined to be 24.26 and $25.30 \mathrm{eV}$ for the $4 d_{5 / 2}$ and $4 d_{3 / 2}$ core levels of $\mathrm{SnS}_{0.9} \mathrm{Se}_{0.1}$ and 24.24 and $25.28 \mathrm{eV}$ for those of SnSe, respectively. An anisotropy in the $a-b$ crystal plane is weak (Tables III and IV).

First, we discuss the results of SnSe. The experimental spectrum for $\mathbf{E} \| \mathbf{a}$ shows structures at 25.30 (A), 25.56 (B),

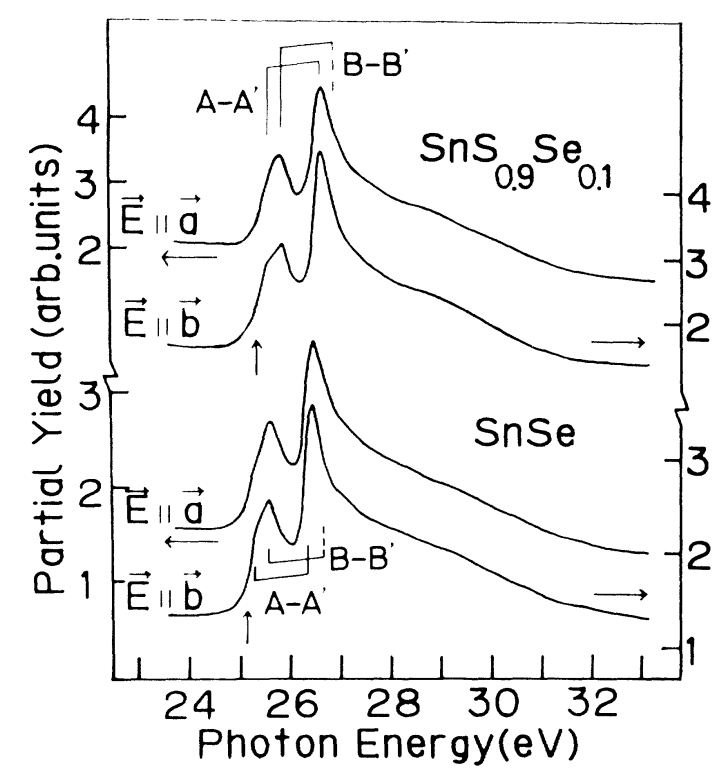

FIG. 7. Partial-yield spectra of $\mathrm{SnS}_{0.9} \mathrm{Se}_{01}$ and $\mathrm{SnSe}$ for $\mathbf{E} \| \mathbf{a}$ and $\mathbf{E} \| \mathbf{b}$ in the $\mathrm{Sn} 4 d$ core excitation region.

and $26.40 \mathrm{eV}\left(\mathrm{A}^{\prime}\right)$. These energies have been accurately determined by calculating the second energy derivatives and are summarized in Table IV. One notices that the splitting energy of $1.08 \mathrm{eV}$ between the structures $\mathrm{A}$ and $\mathrm{A}^{\prime}$ is close to that of the initial $\mathrm{Sn} 4 d$ core level $(1.04 \mathrm{eV})$. For the structure B, the spin-orbit partner $\left(B^{\prime}\right)$ is not clearly seen in the partial-yield spectra. The relative intensities of the A-A $\mathrm{A}^{\prime}$ doublet deviate strongly from the $6: 4$ statistical weight.

The spectrum for $\mathbf{E} \| \mathbf{b}$ is similar to that for $\mathbf{E} \| \mathbf{a}$, except for a slight sharpening of the structure $A^{\prime}$. The sharpening can be attributed to decreasing contribution of $\mathbf{B}^{\prime}$ structure in the spectrum for $\mathbf{E} \| \mathbf{b}$, from comparison of spectral shape among the partial-yield spectra in Fig. 7, the second energy derivatives, and the CIS spectra (which will be shown in Fig. 9). We have already found a similar

TABLE III. Peak energies in partial-yield spectra of $\mathrm{SnS}_{0}{ }_{9} \mathrm{Se}_{(1) 1}$.

\begin{tabular}{|c|c|c|c|c|c|}
\hline & \multirow[t]{2}{*}{ Structure } & \multicolumn{2}{|c|}{ Peak energy } & \multicolumn{2}{|c|}{$\begin{array}{c}\text { Energy above the core } \\
\text { absorption threshold } \\
(\mathrm{eV})\end{array}$} \\
\hline & & $\mathbf{E} \| \mathbf{a}$ & $\mathbf{E} \| \mathbf{b}$ & $\mathbf{E} \| \mathbf{a}$ & $\mathbf{E} \| \mathbf{b}$ \\
\hline \multirow{4}{*}{$\begin{array}{l}\text { Sn } 4 d \text { core } \\
\text { excitation }\end{array}$} & A & $(25.60)$ & 25.59 & 0.2 & 0.2 \\
\hline & B & 25.82 & 25.84 & 0.5 & 0.5 \\
\hline & $\mathrm{A}^{\prime}$ & 26.63 & 26.63 & & \\
\hline & $\mathbf{B}^{\prime}$ & & & & \\
\hline \multirow{2}{*}{$\begin{array}{l}\text { S } 2 p \text { core } \\
\text { excitation }\end{array}$} & $\mathrm{a}$ & 162.5 & 162.5 & 0.7 & 0.7 \\
\hline & $a^{\prime}$ & 163.8 & 163.8 & & \\
\hline
\end{tabular}


TABLE IV. Peak energies in partial-yield spectra of SnSe.

\begin{tabular}{|c|c|c|c|c|c|}
\hline & \multirow[t]{2}{*}{ Structure } & \multicolumn{2}{|c|}{$\begin{array}{c}\text { Peak energy } \\
(\mathrm{eV})\end{array}$} & \multicolumn{2}{|c|}{$\begin{array}{c}\text { Energy above the core } \\
\text { absorption threshold } \\
(\mathrm{eV})\end{array}$} \\
\hline & & $\mathbf{E} \| \mathbf{a}$ & $\mathbf{E} \| \mathbf{b}$ & $\mathbf{E} \| \mathbf{a}$ & $\mathbf{E} \| \mathbf{b}$ \\
\hline \multirow{4}{*}{$\begin{array}{l}\text { Sn } 4 d \text { core } \\
\text { excitation }\end{array}$} & A & $(25.27)$ & 25.30 & 0.1 & 0.2 \\
\hline & B & 25.59 & 25.56 & 0.5 & 0.4 \\
\hline & $\mathrm{A}^{\prime}$ & 26.43 & 26.43 & & \\
\hline & $\mathbf{B}^{\prime}$ & & & & \\
\hline \multirow{3}{*}{$\begin{array}{l}\text { Se } 3 p \text { core } \\
\text { excitation }\end{array}$} & $\mathrm{a}$ & 54.7 & 54.7 & 0.8 & 0.8 \\
\hline & $a^{\prime}$ & 55.6 & 55.6 & & \\
\hline & b & 56.2 & 56.2 & 2.3 & 2.3 \\
\hline
\end{tabular}

situation in GeS and GeSe in Figs. 4 and 5.

The reversal of the relative intensity between the $A$ and $A^{\prime}$ structures indicates the presence of an electron-corehole exchange coupling. Thus, we assign the doublet structure $\mathrm{A}-\mathrm{A}^{\prime}$ to the $\mathrm{Sn} 4 d$ core excitons. For the $\mathrm{B}-\mathrm{B}^{\prime}$ doublet, it will be shown in Sec. III C that the structure B is also attributed to the $\mathrm{Sn} 4 d$ core exciton. The CIS spectrum due to the decay of the core exciton exhibits clear peaks at energies corresponding to the structures $\mathrm{B}$ and $\mathbf{A}-\mathbf{A}^{\prime}$.

Next, we discuss the results of $\mathrm{SnS}_{0.9} \mathrm{Se}_{0.1}$. The partial-yield spectrum for $\mathbf{E} \| \mathbf{b}$ shows a prominent doublet structure $\mathrm{A}-\mathrm{A}^{\prime}$ at $25.60(\mathrm{~A})$ and $26.63 \mathrm{eV}\left(\mathrm{A}^{\prime}\right)$ and a structure $B$ at $25.82 \mathrm{eV}$. The splitting energy between the $\mathrm{A}-\mathrm{A}^{\prime}$ doublet is almost equal to that of $1.04 \mathrm{eV}$ for the $\mathrm{Sn}$ $4 d$ core levels. A reversal of the relative intensities of the doublets is again observed. In comparison with the spectrum for $\mathbf{E} \| \mathbf{a}$, the spectrum shows a slight sharpening of the structure $\mathbf{A}^{\prime}$. The characteristic features of $\mathrm{SnS}_{0.9} \mathrm{Se}_{0.1}$ are essentially the same as those of $\mathrm{SnSe}$, as would be expected from the close similarity between the $\mathrm{Ge} 3 d$ core-yield spectra of $\mathrm{GeS}$ and GeSe. Therefore, we assign the spin-orbit doublets $\mathrm{A}-\mathrm{A}^{\prime}$ and $\mathrm{B}-\mathrm{B}^{\prime}$ to the $\mathrm{Sn} 4 d$ core excitons.

The near-normal-incidence reflectance spectrum of SnS has been measured in the Sn $4 d$ core excitation region with a spectral resolution of $0.2 \mathrm{eV} .^{5}$ This spectrum shows two peaks at 25.65 and $26.50 \mathrm{eV}$ which were interpreted as spin-orbit doublets of the $\mathrm{Sn} 4 d$ core excitons. The reversal of the intensity ratio from the $6: 4$ statistical weight of the Sn $4 d$ core levels was attributed to an electron-core-hole exchange interaction. The large discrepancy of the splitting energies between the reflectance spectrum $(0.85 \mathrm{eV})$ and the core-level photoemission spectrum $(1.08 \mathrm{eV})$ was not explained. In the light of the present results, one notices that the previous assignments in the reflectance spectrum should be corrected. Two peaks in the reflectance spectrum are likely to correspond to the structures $\mathrm{B}$ and $\mathrm{A}^{\prime}$.

\section{Anion core excitation spectra}

Figure 8 shows the partial-yield spectra of $\mathrm{SnS}_{0.9} \mathrm{Se}_{0.1}$ and $\mathrm{SnSe}$ in the $\mathrm{S} 2 p$ and $\mathrm{Se} 3 d$ core excitation region. The spectra reflect the DOS of conduction bands without any sharp peak near the core thresholds shown by vertical arrows $\left(161.8 \mathrm{eV}\right.$ for $\mathrm{SnS}_{0.9} \mathrm{Se}_{0.1}$ and $53.94 \mathrm{eV}$ for $\mathrm{SnSe})$. The spectrum of $\mathrm{SnSe}$ for $\mathbf{E} \| \mathbf{a}$ exhibits three structures at $54.7(\mathrm{a}), 55.6\left(\mathrm{a}^{\prime}\right)$, and $56.2 \mathrm{eV}(\mathrm{b})$. The structures $\mathrm{a}$ and $\mathrm{a}^{\prime}$ can be assigned to a spin-orbit doublet judging from the Se $3 d$ core-level splitting of $0.85 \mathrm{eV}$. The corelevel binding energies are 53.04 and $53.89 \mathrm{eV}$ for the $3 d_{5 / 2}$ and $3 d_{3 / 2}$ components, respectively. As for the structure $b$, its spin-orbit partner is not resolved in the spectrum, probably being obscured by the background. The spectrum for $\mathbf{E} \| \mathbf{b}$ is almost the same as that for $\mathbf{E} \| \mathbf{a}$ with no anisotropy in the $a-b$ crystal plane. The struc-
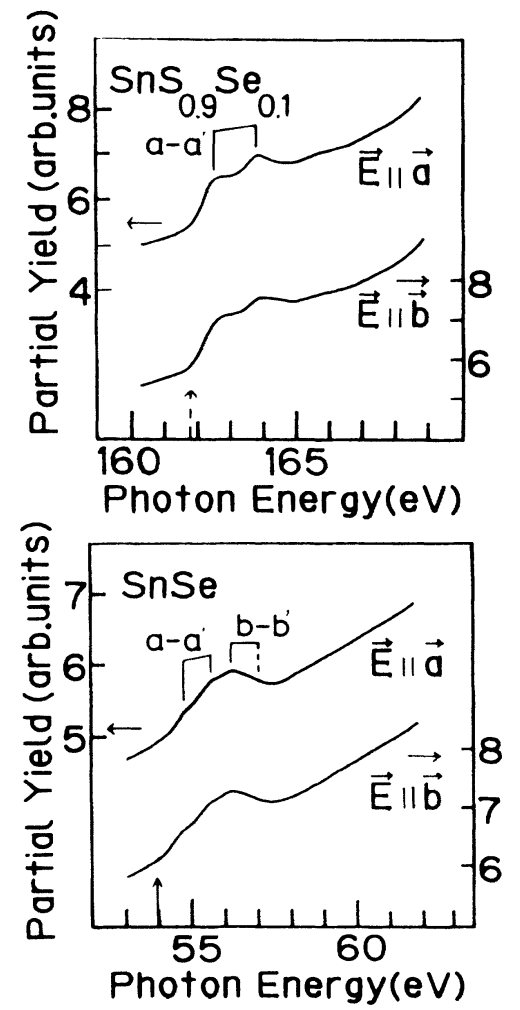

FIG. 8. Partial-yield spectra of $\mathrm{SnS}_{0.9} \mathrm{Se}_{0.1}$ and $\mathrm{SnSe}$ for $\mathbf{E} \| \mathbf{a}$ and $\mathbf{E} \| \mathbf{b}$ in the $\mathrm{S} 2 p$ and Se $3 d$ core excitation regions. 
tures $\mathrm{a}$ and $\mathrm{b}$ are estimated to be located at 0.8 and $2.3 \mathrm{eV}$ above the lowest conduction-band minimum, respectively, from the Se $3 d_{5 / 2}$ core threshold energy of $53.94 \mathrm{eV}$. The energies of the structures $a$ and $b(0.8$ and $2.3 \mathrm{eV})$, band-gap energy [0.9 eV (Ref. 21)], and the binding energy of the first $p$ valence band $(0.6 \mathrm{eV}$ in Fig. 3) imply peaks at 2.3 and $3.8 \mathrm{eV}$ in the $\varepsilon_{2}$ spectra, in agreement with the experimental spectra with a prominent peak at $2.4 \mathrm{eV}$ and a broad peak around $3.5-4 \mathrm{eV} .^{23}$

The spectrum of $\mathrm{SnS}_{0.9} \mathrm{Se}_{0.1}$ for $\mathbf{E} \|$ a shows two broad peaks at 162.5 and $163.8 \mathrm{eV}$. The energy separation of $1.3 \mathrm{eV}$ is almost equal to the $\mathrm{S} 2 p$ core-level splitting of $1.2 \mathrm{eV} .{ }^{17}$ The spectrum for $\mathbf{E} \| \mathbf{b}$ is very similar to that for $\mathbf{E} \|$ a. By taking $161.8 \mathrm{eV}$ as the $\mathrm{S} 2 p$ core threshold energy, ${ }^{18}$ the first peak of the DOS of the conduction bands is evaluated at $0.7 \mathrm{eV}$ above the conduction-band minimum. The optical-absorption measurements have revealed an indirect fundamental gap of $1.1 \mathrm{eV}$ for $\mathrm{SnS}{ }^{22}$ In addition, the $\varepsilon_{2}$ energy-loss spectrum of $\mathrm{SnS}$ has a prominent peak at $2.8 \mathrm{eV}^{4}$ In this case, the initial state is predominantly the first $p$ valence band with $0.8-\mathrm{eV}$ binding energy relative to the top of the valence bands (Fig. 3) so the maximum of the conduction-band DOS is $0.9 \mathrm{eV}$ above the lowest minimum, close to the value of $0.7 \mathrm{eV}$ we estimate in the present experiment.

Here, we compare the cation core-yield spectra in Figs. 4 and 7 with the anion core-yield spectra in Figs. 6 and 8, by adjusting their threshold energies within the same samples. The structures $\mathrm{A}$ due to the cation $d$ core excitons are found to be located $0.5-1.0 \mathrm{eV}$ below the structures a which are assigned to the DOS peaks in the conduction bands. This result suggests that the states of the excited electrons should be described as mixing bands over a width of $\sim 0.5-1.0 \mathrm{eV}$. In addition, the $\mathrm{A}-\mathrm{A}^{\prime}$ and B-B' core excitons separated by $0.21-0.26 \mathrm{eV}$ are mixed with each other. Such mixing has been observed in the Ga $3 d$ core excitons in $\mathrm{GaAs}_{1-x} \mathrm{P}_{x}$ (Ref. 24) and $\mathrm{Al}_{1-x} \mathrm{Ga}_{x}$ As (Ref. 25) crystals.

\section{Decay of cation $d$ core excitons}

We now turn to the CIS spectra measured in the energy region of the cation $d$ core excitation and discuss them in relation with the decay process of the core excitons. Figure 9 shows the photoemission cross sections of the topmost valence-band peaks of $\mathrm{GeS}, \mathrm{GeSe}, \mathrm{SnS}_{0.9} \mathrm{Se}_{0.1}$, and $\mathrm{SnSe}$ single crystals measured as a function of photon energy (CIS spectra). The cross sections exhibit pronounced resonances at the maxima of the corresponding core absorption spectra. The energies of the peaks in the CIS spectra slightly shift with respect to those of the core absorption spectra in Figs. 4 and 7.

The solid curve in Fig. 10 (Ref. 26) represents a fit to the CIS spectrum obtained with Fano profiles:

$$
I \propto \frac{(\varepsilon+q)^{2}}{\varepsilon^{2}+1}, \quad \varepsilon=\frac{1}{\Gamma}\left(\hbar \omega-E_{\mathrm{ex}}\right) .
$$

In Eq. (1), $E_{\mathrm{ex}}, \Gamma$, and $q$ denote the core-exciton excitation energy, the lifetime broadening of the core exciton, and a Fano $q$ factor, respectively. The CIS spectrum is

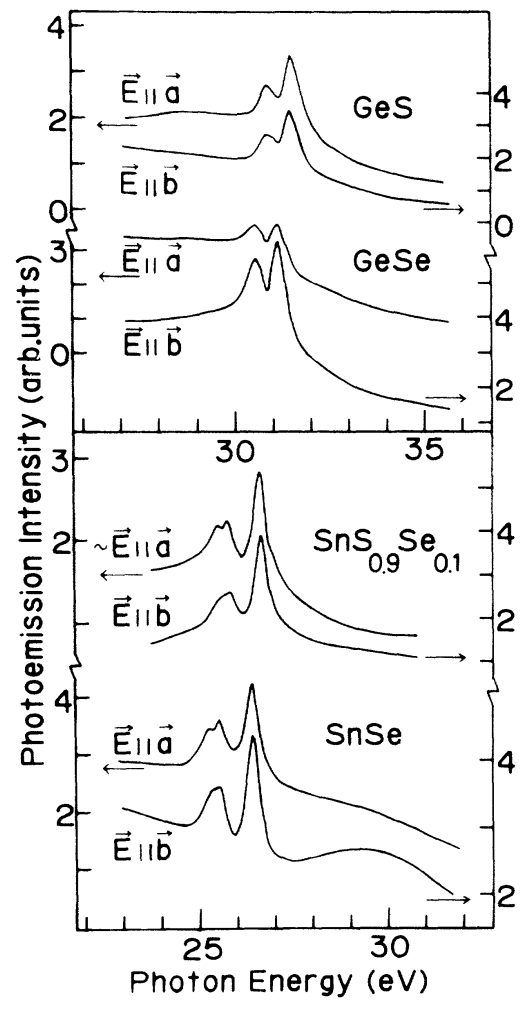

FIG. 9. Photoemission cross sections of the topmost valence-band peaks of $\mathrm{GeS}, \mathrm{GeSe}, \mathrm{SnS}_{0.9} \mathrm{Se}_{0.1}$, and $\mathrm{SnSe}$ single crystals. Each valence band is specified by the binding energy of initial states $\left(E_{l}\right)$ with respect to the valence-band maximum.

well represented by the parameters in Table $\mathrm{V}$, assuming two spin-orbit doublets at the energies of the $A-A^{\prime}$ and B-B' core excitons, linear background, and an instrumental resolution of $100 \mathrm{meV} .^{9}$ The Fano profile describes an interference between the discrete contribution to the valence-band photoemission cross section due to the direct recombination of the core exciton followed by the

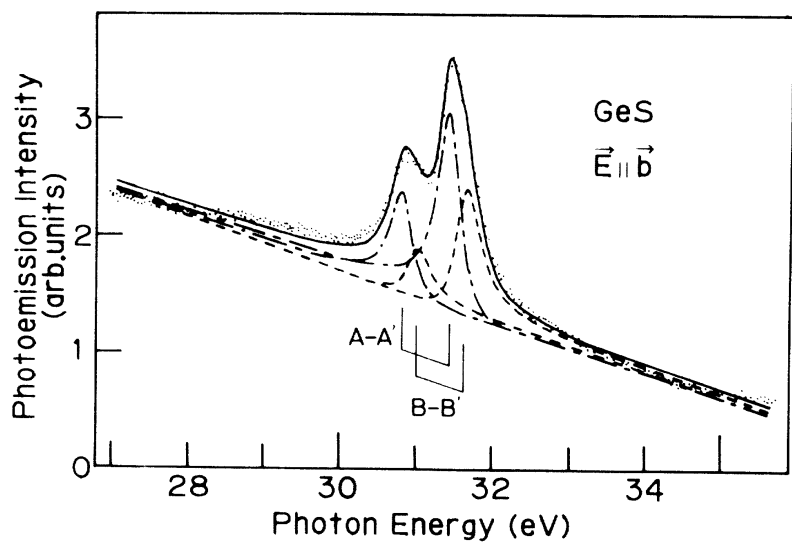

FIG. 10. CIS spectrum of $\mathrm{GeS}(\mathbf{E} \| \mathbf{b})$ for $E_{l}=1.1 \mathrm{eV}$ fitted with four Fano profiles. 
TABLE V. Core-exciton excitation energy, line-shape parameter, lifetime broadening, and intensity.

\begin{tabular}{ccccc}
\hline \hline Structure & $\begin{array}{c}\text { Excitation energy } \\
(\mathrm{eV})\end{array}$ & $q$ & $\begin{array}{c}\Gamma \\
(\mathrm{eV})\end{array}$ & Intensity \\
\hline $\mathrm{A}$ & 30.84 & -7.0 & 0.16 & 0.37 \\
$\mathrm{~A}^{\prime}$ & 31.47 & -7.1 & 0.16 & 0.72 \\
$\mathrm{~B}$ & 31.01 & 6.0 & 0.17 & 0.25 \\
$\mathrm{~B}^{\prime}$ & 31.65 & 5.8 & 0.17 & 0.67 \\
\hline \hline
\end{tabular}

emission of a valence electron, and the usual one-particle excitation. The Fano analysis of the CIS spectra reveals that two pairs of excitons separated by $0.22 \mathrm{eV}$ in the case of $\mathrm{GeS}$ contribute to the core excitation spectrum. The two components are well resolved in the CIS spectra of the Sn compounds (see Fig. 9) and it appears that varying contributions of the two transitions are responsible for the polarization-dependent changes in the CIS and partial-yield spectra. The coherent recombination of core excitons via valence-electron emission constitutes the dominant decay process. The incoherent decay via an $M_{4,5}\left(N_{4,5}\right) V V$ Auger emission is very weak in comparison. A quantitative analysis of the Auger spectra shows that the contribution of the Auger emission to the CIS spectra is very small as shown in the valence-band spectra of Fig. 11.

The difference between excitonic and nonexcitonic core transitions is due to the states near the bottom of conduction bands being primarily derived from cation $p$ states,

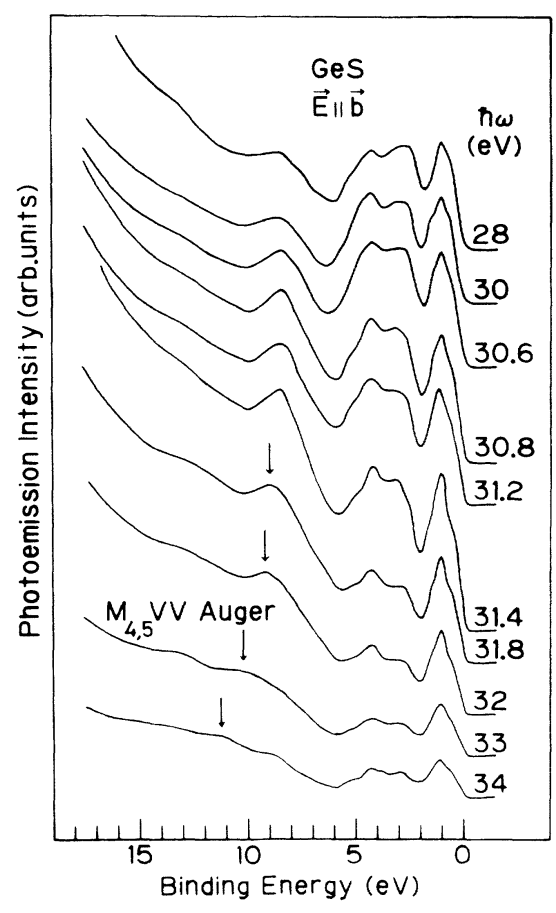

FIG. 11. A series of valence-band photoemission spectra of $\mathrm{GeS}$ for $\mathbf{E} \| \mathbf{b}$ in the Ge $3 d$ core excitation region. The binding energy is defined with respect to the valence-band maximum. whereas the anion $p$ electrons contribute preferentially to the high-DOS features at the top of the valence bands. ${ }^{2,6-8}$ Transitions from cation $d$ states to the bottom of conduction bands are thus dipole-allowed intraatomic transitions with a strong oscillator strength. The quasi-two-dimensional character of the conduction bands as well as the short-range part of the $d$ core hole potential make the excitation more localized and increase the electron-core-hole coupling. The decay of the cation core hole via an $M_{4,5}\left(N_{4,5}\right) \boldsymbol{V} \boldsymbol{V}$ Auger process has the character of an interatomic Auger decay due to the strong anion $p$ contributions of the top part of the valence bands. This accounts for the dominance of the direct recombination channel for the decay of the excitons and thus their comparatively long lifetime. Both factors favor the formation of strong exciton lines at the cation core absorption thresholds.

By the same line of argument, the anion core transitions have the character of charge-transfer excitations with a weak oscillator strength and a low degree of localization. Furthermore, the decay of the core hole via Auger process is rapid. Both factors prevent the formation of stable excitons.

\section{SUMMARY AND CONCLUSIONS}

The conduction-band structures of IV-VI semiconductors with layered orthorhombic structure have been systematically investigated by using synchrotron radiation. The cation $d$ core spectra show intense and sharp doublet structures near the core thresholds, with a weak anisotropy in the $a-b$ crystal plane. These doublets exhibit reversed relative intensity from the initial-state degeneracy and are assigned as $d$ core excitons related to quasi-twodimensional conduction bands with very high DOS. The amount of energy shifts due to excitonic effect is evaluated to be $0.5-1.0 \mathrm{eV}$. The CIS spectra show strong resonance in the cation $d$ core excitation region. The spectra taken for the initial energy below $2 \mathrm{eV}$ with respect to the valence-band maximum are interpreted as an interference between the direct recombination process of the $d$ core excitons and the direct excitation process of the $p$ valence electrons. The narrow width and strong amplitude of the CIS spectra elucidate a long lifetime and a relatively localized character of the core excitons.

The anion $p$ and $d$ core spectra show much broader structures than those in the cation $d$ core spectra. These spectra are assumed to reflect the DOS of the conduction bands. The energies of DOS peaks of the valence and conduction bands derived from the valence-band photoemission and the anion core-yield spectra are related to the energies of the main peaks in the $\varepsilon_{2}$ spectra.

\section{ACKNOWLEDGMENTS}

We are grateful to E. Schönherr and W. Stetter for the crystal growth and to the staff of HASYLAB for their support during the measurements. Discussions with L. Ley in the early stages of this work are acknowledged. One of us (M.T.) would like to thank the Alexander von Humboldt Foundation for provision of financial support. 
${ }^{*}$ Present address: Department of Materials Science, Faculty of Science, Hiroshima University, Higashisenda-machi, Nakaku, Hiroshima 730, Japan.

†Present address: II. Institut für Experimentalphysik, Universität Hamburg, Luruper Chausee 149, D-2000 Hamburg 50, Federal Republic of Germany.

${ }^{\dagger+}$ Present address: Laboratoire de Spectroscopie Electronique, Institut de Recherche sur les Interfaces Solidès, Facultés Universitaires Notre-Dame de la Paix, 61 rue de Bruxelles, B-5000 Namur, Belgium.

${ }^{1}$ P. C. Kenemy, J. Azoulay, M. Cardona, and L. Ley, Nuovo Cimento B 39, 709 (1977).

${ }^{2}$ T. Granke and L. Ley, Phys. Rev. B 16, 832 (1977).

${ }^{3}$ V. Chab and I. Bartos, Phys. Status Solidi B 121, 301 (1984).

${ }^{4}$ R. Eymard and A. Otto, Phys. Rev. B 16, 1616 (1977).

${ }^{5}$ A. Otto, L. Ley, J. Azoulay, T. Granke, R. Eymard, W. Braun, and M. Cardona, Phys. Rev. B 16, 4429 (1977).

${ }^{6} \mathrm{G}$. Valiukonis, F. M. Gashimade, D. A. Guseinova, G. Krivaite, A. M. Kulibekov, G. S. Orudzhev, and A. Sileika, Phys. Status Solidi B 117, 81 (1983); the authors chose a rather special notation for the symmetry points and lines in the Brillouin zone of the space group $D_{2 h}^{16}$ characteristic of the family of the $A^{\mathrm{IV}} B^{\mathrm{VI}}$ compounds with a simple orthorhombic lattice.

${ }^{7}$ A. W. Parke and G. P. Srivastava, Phys. Status Solidi B 101, K31 (1980).

${ }^{8}$ R. Car, G. Ciucci, and L. Quartapelle, Phys. Status Solidi B 86, 471 (1978).

${ }^{9}$ R. L. Johnson and J. Reichardt, Nucl. Instrum. Methods 208, 791 (1983).

${ }^{10}$ E. Schönherr, J. Cryst. Growth 57, 493 (1982).

${ }^{11}$ H. Asahina, K. Shindo, and A. Morita, J. Phys. Soc. Jpn. 51, 1193 (1982).

12J. D. Wiley, D. Thomas, E. Schonherr, and A. Breitschwerdt, J. Phys. Chem. Solids 41, 801 (1980).

${ }^{13}$ W. Gudat and C. Kunz, Phys. Rev. Lett. 29, 169 (1972).

${ }^{14} \mathrm{~A}$. M. El-Korashy, in Proceedings of the 14th International Conference on the Physics of Semiconductors, Edinburgh, 1978, edited by B. L. H. Wilson (The Institute of Physics, Bristol, 1979), p. 817.

${ }^{15}$ Y. Onodera and Y. Toyozawa, J. Phys. Soc. Jpn. 22, 833
(1967).

${ }^{16}$ R. L. Johnson, J. H. Fock, L. Ley, and M. Cardona, in Proceedings of the 17th International Conference on the Physics of Semiconductors, San Francisco, 1984, edited by J. D. Chadi and W. A. Harrison (Springer, New York, 1985), p. 1239.

${ }^{17}$ Photoemission in Solids II, edited by L. Ley and M. Cardona (Springer, Berlin, 1979), p. 375.

${ }^{18}$ Energies of the $\mathrm{Ge} 3 d, \mathrm{Sn} 4 d$, and Se $3 d$ core absorption thresholds have been estimated from the sum of band-gap energy and core-level binding energy as mentioned in Sec. III A 1. For the S $2 p$ core thresholds of $\mathrm{GeS}$ and $\mathrm{SnS}_{0}{ }_{9} \mathrm{Se}_{0.1}$, these energy positions have been evaluated directly from the core-yield spectra by linearly extrapolating the leading edge of the absorption to the smooth background, since photoemission experiments for the $\mathrm{S} 2 p$ core levels are difficult due to the limited monochromator energy range. One notices that the energies of anion core (Se $4 d$ ) thresholds of GeSe and SnSe obtained from such a direct procedure coincide with those estimated from the sum of two energies mentioned above.

${ }^{19}$ S. Logothetidis, L. Viña, and M. Cardona, Phys. Rev. B 31, 2180 (1985).

${ }^{20}$ A. W. Parke and G. P. Srivastava, Phys. Status Solidi B 101, K31 (1980).

${ }^{21}$ W. Albers, C. Haas, H. Ober, G. R. Shodder, and J. D. Wassher, J. Phys. Chem. Solids 23, 215 (1962); A. G. Mikolaichuk and D. M. Frei, Fiz. Tverd. Tela (Leningrad) 11, 2520 (1969).

${ }^{22}$ A. P. Lambros, D. Geraleas, and N. A. Economou, J. Phys. Chem. Solids 35, 537 (1974).

${ }^{23}$ S. Logothetidis and H. M. Polatoglou, Phys. Rev. B 36, 7491 (1987).

${ }^{24}$ S. M. Kelso, D. E. Aspnes, C. G. Olson, D. W. Lynch, and D. Finn, Phys. Rev. Lett. 45, 1032 (1980).

${ }^{25}$ M. Taniguchi, S. Suga, S. Shin, K. Inoue, M. Seki, and H. Kanzaki, Solid State Commun. 44, 1079 (1982).

${ }^{26} \mathrm{M}$. Taniguchi, J. Ghijsen, R. L. Johnson, and L. Ley, in Proceedings of the 18th International Conference on the Physics of Semiconductors, Stockholm, 1986, edited by O. Engström (World Scientific, Singapore, 1987), p. 1799. 